\title{
Utilizing Mechanical Strain to Mitigate the Intrinsic Loss Mechanisms in Oscillating Metal Nanowires
}

\author{
Sung Youb Kim ${ }^{1,2}$ and Harold S. Park ${ }^{1,2, *}$ \\ ${ }^{1}$ Department of Mechanical Engineering, University of Colorado, Boulder, Colorado 80309, USA \\ ${ }^{2}$ DARPA Center for Integrated Micro/Nano-Electromechanical Transducers (iMINT), University of Colorado, \\ Boulder, Colorado 80309, USA
}

(Received 8 August 2008; published 21 November 2008)

\begin{abstract}
We utilize classical molecular dynamics to study energy dissipation (the $Q$ factors) of doubly clamped copper nanowire nanoresonators undergoing flexural oscillations. We find that the application of tensile strain effectively mitigates both the intrinsic surface and thermal losses, with improvements in $Q$ by a factor of 3-10 across a range of operating temperatures. We also find that the nanowire $Q$ factors are not dependent on the surface area to volume ratio, but instead their aspect ratio, and that the $Q$ factors exhibit a $1 / T^{0.70}$ dependence on the temperature $T$ that is independent of strain.
\end{abstract}

DOI: 10.1103/PhysRevLett.101.215502

Over the past decade, driven by their remarkable physical properties, nanowires have drawn considerable interest from the scientific community. These novel physical properties have motivated the development of novel nanowirebased nanoelectromechanical systems (NEMS), which have been proposed for chemical and biological sensing [1], force and pressure sensors [2], high-frequency resonators for next-generation wireless devices [3], and many other applications [4].

Operationally, these nanowire-based NEMS utilize the nanowire as a resonating beam, where the nanowire oscillates continuously at or near its resonant frequency and where the desired change in local environment, i.e., force, pressure, or mass, can be detected by changes in the resonant frequency of the nanowire. Therefore, the key performance measure for nanowires is their quality $(Q)$ factor, which measures the energy dissipated per vibrational cycle. A higher $Q$ factor is critical to NEMS device performance and reliability as it implies less energy dissipation per vibrational cycle, which enables the nanowire to extend its operational lifetime by performing near optimal capacity for a longer period of time. Furthermore, for these sensing applications, the sensing resolution is inversely proportion to the $Q$ factor; the mass sensitivity $\delta m[4]$ is defined as

$$
\delta m \approx\left(\frac{2 M_{\mathrm{eff}}}{Q}\right) 10^{-\mathrm{DR} / 20},
$$

where DR is the dynamic range and $M_{\text {eff }}$ is the effective mass of the nanowire. From (1), it is evident that low $Q$ factors are the key limiting factor to the development of ultrasmall, highly sensitive, and reliable NEMS.

Nanowires can dissipate energy and thereby lower their $Q$ factors through both intrinsic and extrinsic means; extrinsic losses occur due to interactions with the surrounding environment, typically through support or clamping losses and through gas or air damping [5], while intrinsic losses arise due to processes occurring within the nanowire itself,
PACS numbers: 62.20. $-\mathrm{x}, 62.23 . \mathrm{Hj}, 62.25 . \mathrm{Fg}, 62.25 . \mathrm{Jk}$

for example, due to surface-driven losses [6,7] and thermoelastic damping $[5,8,9]$. Various researchers have shown $[6,7]$ that the surface loss increases in essentially a linear fashion with increasing surface area to volume ratio or decreasing nanowire size. Despite the experimental evidence demonstrating the importance of surface and thermal losses in degrading the nanowire $Q$ factors, it is still not understood how these losses can be mitigated. One approach that has shown promise is to passivate the dangling bonds at the nanowire surfaces, for example, passivating $\mathrm{Si}$ surfaces with hydrogen [7]. Other researchers have had success increasing the $Q$ factors of $\mathrm{SiN}, \mathrm{Si}$, and $\mathrm{SiC}$ nanowires through the application of tensile stress $[10,11]$; however, the $100 \mathrm{~nm}$ cross section nanowires utilized in those works indicate size scales at which surface losses may not dominate the $Q$-factor degradation. Therefore, a systematic means of combatting the intrinsic dissipation mechanisms in nanowires remains a significant and open research topic.

In the present work, we view surface losses as occurring as a result of the different vibrational frequencies of surface atoms and bulk atoms; surface atoms vibrate at a different frequency due to their different stiffness as compared to bulk atoms [12]. Passivation of undercoordinated silicon surface atoms with hydrogen, as employed by Yang, Ono, and Esashi [7], is likely effective in enhancing the nanowire $Q$ factors because the bonding environment, and thus the elastic stiffness of the surface atoms, becomes more bulklike. As the nanowire size decreases, the nanowire vibrational properties become strongly impacted by the noncoherent oscillations of the bulk and surface atoms, leading to $Q$-factor reduction through surface losses. The hypothesis advanced in the present work is that tensile mechanical strain can be employed to reduce this stiffness difference between bulk and surface atoms, thereby enhancing the nanowire $Q$ factors.

Therefore, we present in this work the results of classical molecular dynamics (MD) calculations supporting the idea 
that the application of mechanical strain is an effective way to mitigate the intrinsic $Q$ degrading mechanisms in oscillating copper nanowires; we neglect extrinsic damping effects by assuming the nanowires oscillate in vacuum and by fixing the support atoms. The MD simulations were performed using an embedded-atom-type potential for copper developed by Cai and Ye [13]. First, $\langle 100\rangle$ copper nanowires with $\{100\}$ transverse surfaces with square cross sections of length ranging from 1.45 to $2.9 \mathrm{~nm}$ with aspect ratios ranging from 7 to 15 were created with the atomic positions placed according to the fcc lattice with the bulk lattice spacing for copper $(3.615 \AA)$; while $\langle 100\rangle$ nanowires were utilized in the present work, the findings should be applicable to metal nanowires with other axial orientations. The nanowires were then equilibrated at a specified temperature ranging from 1 to $300 \mathrm{~K}$ for 100000 steps at a time step of 5 femtoseconds with both ends fixed using a constant temperature $(N V T)$ NoseHoover thermostat [14]; this state specifies the 0\% strain state in Figs. 2 and 4. After the initial thermal equilibration, one end of the nanowire was either stretched or compressed to a specified state of tensile or compressive strain, after which the nanowire was equilibrated again for 100000 steps with both ends fixed at the same temperature. After the second thermal equilibration, a sinusoidal transverse velocity field was applied to the doubly fixed nanowire resulting in an initial displacement that was about 3\% of the nanowire length and that causes the nanowire to oscillate; the applied velocity field increased the total system potential energy (PE) at most by $0.1 \%$ to ensure that nonlinear modes of vibration would not be excited. The oscillation of the nanowire was then simulated using a constant energy ( $N V E$ ) ensemble for a total of 2500 picoseconds, corresponding to nearly 100 complete cycles of oscillation.

Similarly to Jiang et al. [15], we illustrate in Fig. 1 the external energy (EE) time history of an oscillating $10.85 \times$ $1.45 \times 1.45 \mathrm{~nm}$ copper nanowire, in both cases at $10 \mathrm{~K}$ but at different amounts of strain, i.e., $2.5 \%$ compressive and $1.5 \%$ tensile strain; the EE is defined as the difference of the PE before and after the transverse velocity is applied to the nanowire. We note two critical trends of interest. First, it is apparent for both states of strain that the EE decreases in a linear fashion as a function of time, which indicates that the oscillation does not excite nonlinear modes of vibration. We note that, because the nanowire oscillations were simulated in an energy-preserving $N V E$ ensemble, the EE that is lost must be converted to kinetic energy. Because the EE decrease is linear with each nanowire oscillation cycle, at the end of $n$ cycles, the final EE $\left(\mathrm{EE}_{n}\right)$ is related to the initial $\mathrm{EE}\left(\mathrm{EE}_{0}\right)$ as $\mathrm{EE}_{n}=\mathrm{EE}_{0}(1-$ $2 \pi / Q)^{n}$ [15]; the $Q$ factors presented in this work were calculated using this relationship. Second, it is apparent that there is considerably more EE dissipation in Fig. 1 at $2.5 \%$ compressive strain than at $1.5 \%$ tensile strain; due to the preceding relationship between the $Q$ factor and $\mathrm{EE}$, the $Q$ factor of the nanowire at $1.5 \%$ tensile strain will therefore be larger than the $Q$ factor at $2.5 \%$ compressive strain.

We quantify in Fig. 2 the amount of $Q$-factor increase that is possible in straining the $10.85 \times 1.45 \times 1.45 \mathrm{~nm}$ copper nanowire between 3\% compressive strain and $4 \%$ tensile strain for temperatures ranging from 1 to $300 \mathrm{~K}$. There are three key implications made by Fig. 2, which we now discuss. First, it is evident that the $Q$ factors of doubly fixed nanowire-based NEMS can be tuned across a range of values; in particular, the application of tensile strain is seen to be highly beneficial to improving the nanowire $Q$ factor, whereby the maximum $Q$ factor, which occurs around $1.5 \%$ tensile strain at all temperatures, is between 4.5 10 times the minimum $Q$ factor, which occurs around 3\% compressive strain at all temperatures.

Second, Fig. 2 demonstrates that tensile strain can also be utilized to combat thermally driven losses in oscillating nanowires. We find that the maximum gain in the $Q$ factor is consistently at least a factor of 4.5 across a range of temperatures from 1 to $300 \mathrm{~K}$ when the strain on the doubly fixed nanowires is varied between $3 \%$ compressive and $1.5 \%$ tensile strain.
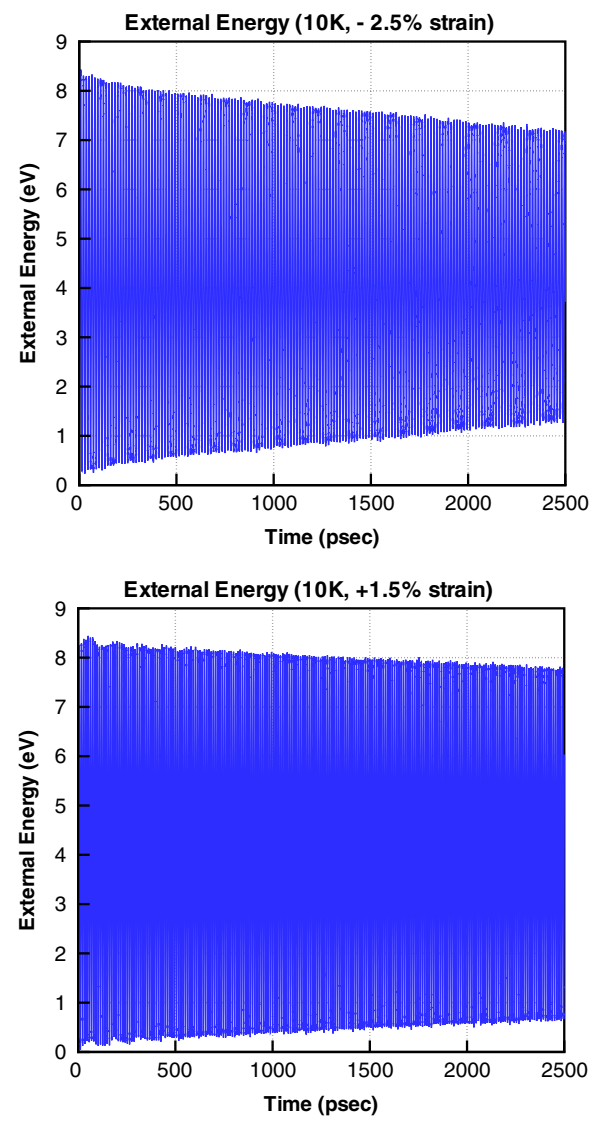

FIG. 1 (color online). Top: External energy time history of oscillating $10.85 \times 1.45 \times 1.45 \mathrm{~nm}$ copper nanowire at $10 \mathrm{~K}$, $2.5 \%$ compressive strain. Bottom: External energy time history of oscillating $10.85 \times 1.45 \times 1.45 \mathrm{~nm}$ copper nanowire at $10 \mathrm{~K}$, $1.5 \%$ tensile strain. 


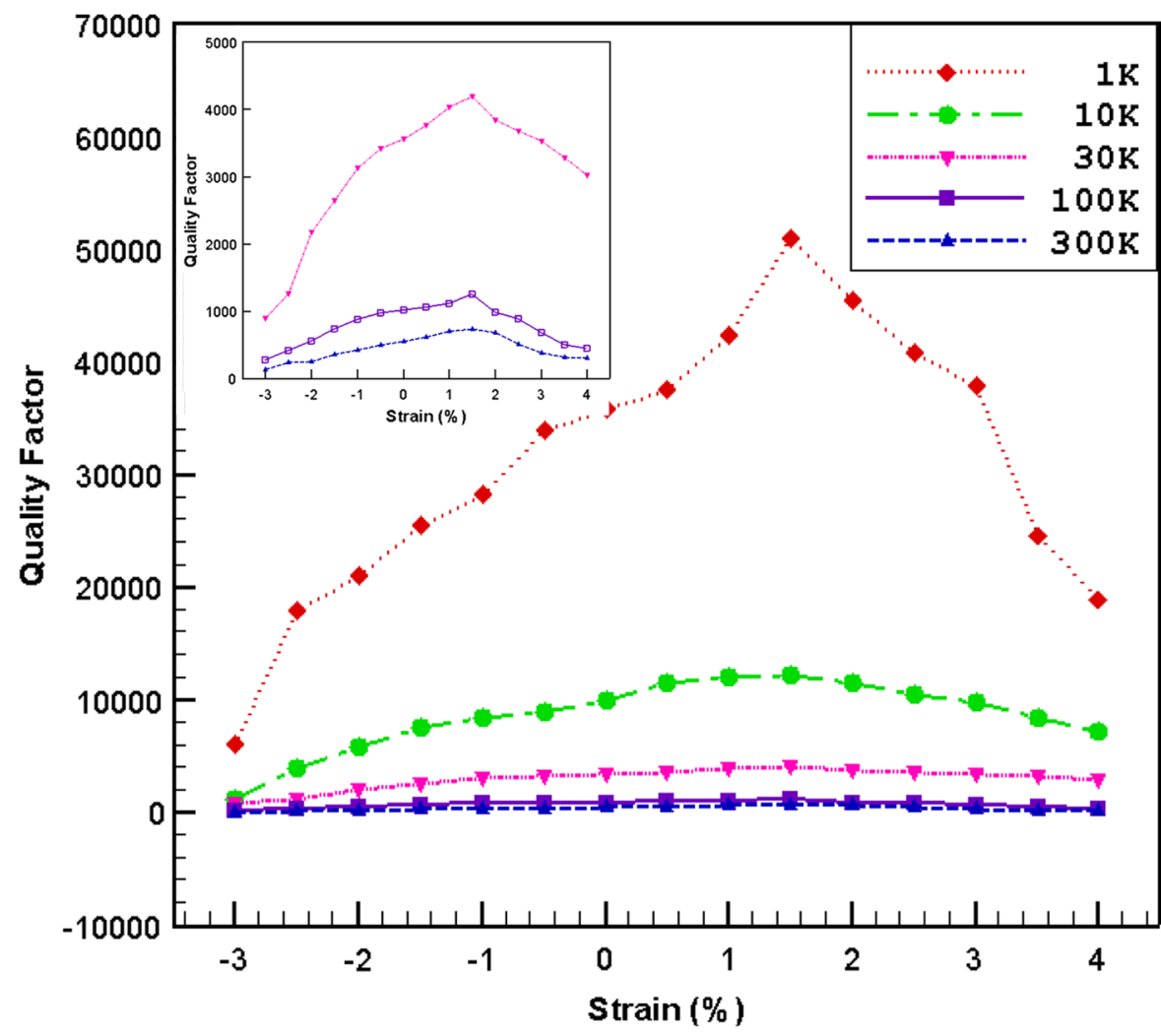

FIG. 2 (color online). $Q$ factors as a function of strain for $10.85 \times 1.45 \times$ $1.45 \mathrm{~nm}$ copper nanowire at various temperatures. Inset: $Q$ factors at 30,100, and $300 \mathrm{~K}$.

Third, because Fig. 2 shows that compressive strains lead to significantly lower $Q$ factors, it has significant implications for the $Q$ factors of nanowires depending on the boundary condition utilized; this is because metal nanowires that have fixed/free boundary conditions are known to contract dramatically in compression due to the existence of nanoscale surface stresses [16]. For the $1.45 \mathrm{~nm}$ cross section copper nanowire in Fig. 2, the free end elastically contracts by slightly more than $3 \%$ in compression due to the surface stresses. Therefore, Fig. 2 indicates that the intrinsic $Q$ factors of fixed/free metal nanowire-based NEMS should be 4.5-10 times lower than the $Q$ factors of fixed/fixed nanowires for cross sectional sizes smaller than $2 \mathrm{~nm}$. However, due to the small sizes of the fixed/free nanowires considered in this work, they were found to undergo structural reorientations resulting in 30\% compressive strain due to thermal fluctuations as has been found previously for metal nanowires leading to shape memory and pseudoelastic behavior [16]. However, the boundary condition effect is predicted to theoretically exist in larger nanowires where the structural reorientations do not occur; $10 \mathrm{~nm}$ cross section metal nanowires are predicted to compress more than $1 \%$ due to surface stresses [17], which would imply a gain of 2-3 times in the $Q$ factor simply by using fixed/fixed boundary conditions as compared to fixed/free.

To further quantify the dependence of the $Q$ factor on temperature, we plot in Fig. 3 the $Q$ factor for various states of strain against the nanowire temperature. From Fig. 3, similar to previous works where the $Q$ factor vs temperature relationship of carbon nanotubes [15] and silicon nanowires [18] were found, we determine the relationship between $Q$ and the temperature $T$ to be $Q \sim$ $1 / T^{0.7}$. In contrast, the exponent on the temperature was found to be 0.25 for silicon by Mohanty et al. [18] and 0.36 for the nanotubes by Jiang et al. [15], indicating that metal nanowires exhibit a greater reduction in $Q$ with increasing temperature than do nanotubes or semiconducting nanowires. It is also interesting that, while mechanical strain was shown in Fig. 2 to be effective in elevating the $Q$ factors for various temperatures, the rate of $Q$ factor decrease remains essentially constant as shown in Fig. 3

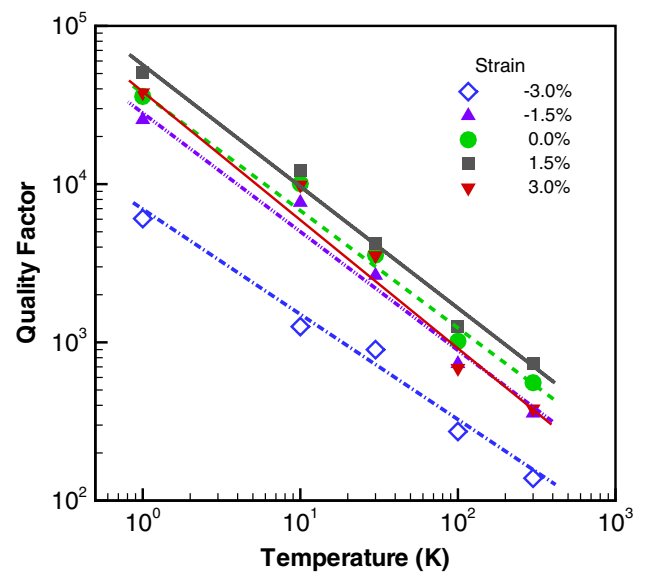

FIG. 3 (color online). Temperature dependence of the $Q$ factors for $10.85 \times 1.45 \times 1.45$ nanowires as a function of applied uniaxial strain. 


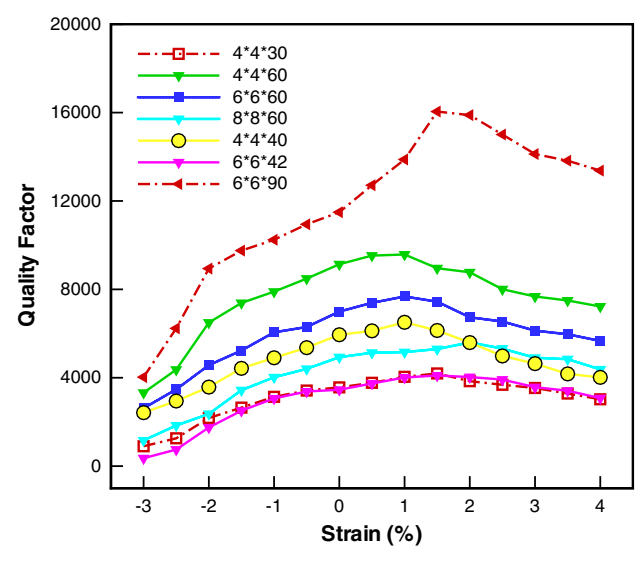

FIG. 4 (color online). Effect of mechanical strain on the $Q$ factors of copper nanowires (dimensions in CLUs) of various sizes at $30 \mathrm{~K}$.

when the temperature is increased for a given nanowire state of strain.

We also studied nanowires with larger cross sectional sizes, to determine whether applied mechanical strain can be used to enhance the $Q$ factors of larger nanowires. The results for nanowires oscillating at $30 \mathrm{~K}$ with cross sectional sizes ranging from 4 to 8 cubic lattice units (CLUs), where $1 \mathrm{CLU}=3.615 \AA$, the lattice constant of copper, and with lengths ranging from 30 to 60 CLUs are shown in Fig. 4.

In doing so, we find that the $Q$ factors are independent of the surface area to volume ratio; this finding differs from the previously noted surface area to volume dependent on the $Q$-factor degradation, though it is important to note that most experiments detailing surface-driven $Q$ losses have been performed on nanowires with cross sectional sizes greater than $100 \mathrm{~nm}$ [6]. Instead, we find that there is a cross sectional (CS) and aspect ratio (AR) dependence for $Q$. For example, $Q$ increases with increasing AR (or $d e$ creasing surface area to volume ratio) for a constant CS; for the 4 CLU CS nanowires, $Q$ increases from 4200 to 9600 as the AR doubles, while for the 6 CLU CS nanowires, $Q$ increases from 4100 to 16000 as the AR increases from 7 to 15 . However, for a given AR, $Q$ increases as the CS increases, which is in agreement with experiments in that larger nanowires exhibit less surface loss. For example, when the $\mathrm{AR}=10, Q$ increases from 6500 for a $4 \mathrm{CLU}$ CS to 7700 for a $6 \mathrm{CLU}$ CS, and when the AR = 15, $Q$ increases from 9500 for a 4 CLU CS to 16000 for a 6 CLU CS. Finally, we note that, for the larger 6 CLU cross section nanowires, the gain in $Q$ factor again ranges from about 3 to 10 times with the application of tensile strain.

In conclusion, we have utilized classical molecular dynamics simulations to determine that the application of tensile mechanical strain effectively mitigates the intrinsic surface and thermally driven loss mechanisms in oscillating copper nanowires. Furthermore, the current findings can be experimentally validated, as recent experiments have indicated the ability to apply axial strain to nanowires
[10]; we note again that experiments have demonstrated the utility of mechanical stress in improving the $Q$ factors of $100 \mathrm{~nm}$ cross section $\mathrm{SiN}, \mathrm{Si}$, and $\mathrm{SiC}$ nanowires $[10,11]$. We also find that the nanowire $Q$ factors are dependent on the aspect ratio, and not the surface area to volume ratio, and that the type of boundary condition applied to the nanowires should strongly impact the available $Q$ factor, where fixed/fixed nanowires are expected to have higher intrinsic $Q$ factors than fixed/free nanowires of the same size. These findings should benefit the ongoing thrust within the nanotechnology community to design high $Q$-factor nanowire-based NEMS.

The studies conducted by the authors from the University of Colorado-Boulder are supported by the DARPA Center on Nanoscale Science and Technology for Integrated Micro/Nano-Electromechanical Transducers (iMINT) funded by the DARPA N/MEMS S\&T Fundamentals Program (HR0011-06-1-0048). H. S. P. also acknowledges NSF Grant No. CMMI-0750395.

*harold.park@colorado.edu

[1] B. Ilic, Y. Yang, K. Aubin, R. Reichenbach, S. Krylov, and H. G. Craighead, Nano Lett. 5, 925 (2005).

[2] T. D. Stowe, K. Yasumura, T. W. Kenny, D. Botkin, K. Wago, and D. Rugar, Appl. Phys. Lett. 71, 288 (1997).

[3] H. G. Craighead, Science 290, 1532 (2000).

[4] K. L. Ekinci and M.L. Roukes, Rev. Sci. Instrum. 76, 061101 (2005).

[5] M. Chu, R.E. Rudd, and M.P. Blencowe, arXiv:org p.0705.0015v1.

[6] D. W. Carr, S. Evoy, L. Sekaric, H. G. Craighead, and J. M. Parpia, Appl. Phys. Lett. 75, 920 (1999).

[7] J. Yang, T. Ono, and M. Esashi, J. Vac. Sci. Technol. B 19, 551 (2001).

[8] S. Evoy, A. Olkhovets, L. Sekaric, J. M. Parpia, H. G. Craighead, and D. W. Carr, Appl. Phys. Lett. 77, 2397 (2000).

[9] R. Lifshitz and M.L. Roukes, Phys. Rev. B 61, 5600 (2000).

[10] S. S. Verbridge, D. F. Shapiro, H. G. Craighead, and J. M. Parpia, Nano Lett. 7, 1728 (2007).

[11] V. Cimalla, C. Foerster, F. Will, K. Tonisch, K. Brueckner, R. Stephan, M. E. Hein, O. Ambacher, and E. Aperathitis, Appl. Phys. Lett. 88, 253501 (2006).

[12] L. G. Zhou and H. Huang, Appl. Phys. Lett. 84, 1940 (2004).

[13] J. Cai and Y. Y. Ye, Phys. Rev. B 54, 8398 (1996).

[14] W. G. Hoover, Phys. Rev. A 31, 1695 (1985).

[15] H. Jiang, M. F. Yu, B. Liu, and Y. Huang, Phys. Rev. Lett. 93, 185501 (2004).

[16] H. S. Park, K. Gall, and J. A. Zimmerman, Phys. Rev. Lett. 95, 255504 (2005).

[17] H. S. Park and P.A. Klein, Phys. Rev. B 75, 085408 (2007).

[18] P. Mohanty, D. A. Harrington, K. L. Ekinci, Y. T. Yang, M. J. Murphy, and M. L. Roukes, Phys. Rev. B 66, 085416 (2002). 